\title{
CONFIGURATION-LIKE SPACES AND THE BORSUK-ULAM THEOREM
}

\author{
FRED COHEN AND EWING L. LUSK
}

\begin{abstract}
Some extensions of the classical Borsuk-Ulam Theorem are proved by computing a bound on the homology of certain spaces similar to configuration spaces. The Bourgin-Yang Theorem and a generalization due to Munkholm are special cases of these results.
\end{abstract}

1. Introduction. The purpose of this paper is to extend and unify several generalizations of the Borsuk-Ulam Theorem. Let $\pi_{p}$ denote the cyclic group of prime order $p$ and let $X$ be a pathwise connected Hausdorff space on which $\pi_{p}$ acts freely. Suppose that $M$ is some fixed manifold and that $f: X \rightarrow M$ is any map. We are interested in conditions on $X$, depending on $M$ but not on $f$, which are sufficient to insure that a certain number of points in some orbit are sent to the same point in $M$ by $f$. Specifically, let $\sigma$ denote the generator of $\pi_{p}$ and define

$$
\begin{aligned}
A(f, q)=\left\{x \in X \mid \text { there exist } i_{1}, i_{2}, \ldots, i_{q} \text { with } 0 \leqq i_{1}<i_{2}\right. \\
\left.\qquad \cdots<i_{q}<p \text { and } f\left(\sigma^{i_{1}} x\right)=f\left(\sigma^{i_{2}} x\right)=\cdots=f\left(\sigma^{i_{q}} x\right)\right\} .
\end{aligned}
$$

In the case $M=R^{n}$, we prove the following, in which $\operatorname{dim} A$ denotes the covering dimension of $A$, and all cohomology is taken with $\mathbf{Z}_{p}$ coefficients unless otherwise stated.

THEOREM 1. If $H^{i}(X)=0$ for $0<i<(n-1)(p-1)+q-1$ and $q$ $\geqq \frac{1}{2}(p+1)$ or $q=2$, then $A(f, q) \neq \varnothing$.

THEOREM 2. If $X$ is a $\mathbf{Z}_{p}$-orientable m-manifold and $H^{i}(X)=0$ for $0<i$ $<(n-1)(p-1)+q-1$ and $q \geqq \frac{1}{2}(p+1)$ or $q=2$, then $\operatorname{dim} A \geqq m$ $-(n-1)(p-1)-q+1$.

Special cases of these theorems are known:

1. The classical Borsuk-Ulam Theorem is Theorem 1 with $X=S^{n}$ and $q$ $=p=2[1]$.

2. The "mod $p$ Bourgin-Yang Theorem" of Munkholm is Theorem 2 with $q=p$ and $X$ a mod $p$ homology $m$-sphere [6]. For this special case the proof given below is much simpler than Munkholm's.

3. The case $q=2$ of Theorem 1 appears in [3].

Theorems 1 and 2 are actually special cases of a more general theorem, in which $R^{n}$ is replaced by an arbitrary manifold $M$. That is, for each $M$ and

Received by the editors September 10, 1974 and, in revised form, January 20, 1975.

AMS (MOS) subject classifications (1970). Primary 55C20, 55C35, 54H25.

() American Mathematical Society 1976 
$q \leqq p$ there is a number $N(M, p, q)$ (defined below) such that for $f: X \rightarrow M$ we have:

Theorem 3. If $H^{i}(X)=0$ for $0<i \leqq N(M, p, q)$, then $A(f, q) \neq \varnothing$. If in addition we assume that $X$ is a $\mathbf{Z}_{p}$-orientable m-manifold, then $\operatorname{dim} A(f, q)$ $\geqq m-N(M, p, q)-1$.

To define the numbers $N(M, p, q)$, consider the subspace $G(M, p, q)$ of $(M)^{p}$ consisting of the $p$-tuples in which no $q$ coordinates coincide. More precisely, $G(M, p, q)=\left\{\left(x_{1}, \ldots, x_{p}\right) \mid\right.$ for any $\left\{x_{i_{1}}, \ldots, x_{i_{q}}\right\}$ with $0<i_{1}$

$$
\left.<\cdots<i_{q} \leqq p \text {, at least } 2 \text { of the } x_{i_{j}} \text { 's are different }\right\} .
$$

Note that $G(M, p, q) \subset G(M, p, q+1), G(M, p, p)=(M)^{p}-\Delta_{M}$, and $G(M, p, 2)$ is the Fadell-Neuwirth configuration space [5]. The group $\pi_{p}$ acts freely on $G(M, p, q)$ by cyclic permutation of coordinates, and the inclusions $G(M, p, q) \subset G(M, p, p)$ are equivariant. For some large $n, G(M, p, q)$ embeds in $G\left(R^{n}, p, q\right)$ via the embedding of $M$ in $R^{n}$. Define

$$
G\left(R^{\infty}, p, q\right)=\lim _{\rightarrow} G\left(R^{n}, p, q\right) .
$$

Proposition. $G\left(R^{\infty}, p, p\right)$ is a free $\pi_{p}$-space with trivial homotopy groups and hence $G\left(R^{\infty}, p, p\right) / \pi_{p}$ is $a K\left(\pi_{p}, 1\right)$.

Proof. Since

$$
H_{*}\left(G\left(R^{\infty}, p, p\right) ; \mathbf{Z}\right)=H_{*}\left(\lim _{\rightarrow} G\left(R^{n}, p, p\right) ; \mathbf{Z}\right)=\lim _{\rightarrow} H_{*}\left(G\left(R^{n}, p, p\right) ; \mathbf{Z}\right)
$$

and $G\left(R^{n}, p, p\right) \simeq S^{n(p-1)-1}, G\left(R^{\infty}, p, p\right)$ has trivial homology groups. Since $G\left(R^{\infty}, p, p\right)$ is simply connected, the result follows from the Hurewicz Theorem.

Definition of $N(M, p, q)$. Let $\phi$ be an equivariant embedding of $G(M, p, q)$ in $G\left(R^{\infty}, p, p\right)$. Recall that $H^{i} K\left(\pi_{p}, 1\right)=\mathbf{Z}_{p}$ for all $i$ and define $N(M, p, q)$ to be the largest $N$ such that $\phi^{*}$ is not the zero homomorphism. We have not calculated $N(M, p, q)$ for $M \neq R^{n}$ except for the case $q=2$ (see [4]). When $M=R^{n}$, it is sufficient to calculate the first nonvanishing homology class in a certain union of spheres. This we do in $\S 3$. The result is:

THEOREM 4. $N\left(R^{n}, p, q\right) \leqq(n-1)(p-1)+q-2$ if $q \geqq \frac{1}{2}(p+1)$ or $q$ $=2$.

2. Proofs of Theorems 1, 2, and 3. We prove Theorem 3. Theorems 1 and 2 follow immediately from Theorems 3 and 4. Let $\sigma$ be the generator of $\pi_{p}$ and define $\psi: X \rightarrow(M)^{p}$ by $\psi(x)=\left(f(x), f(\sigma x), \ldots, f\left(\sigma^{p-1} x\right)\right)$. If $A(f, q)=\varnothing$ then $\psi$ is an equivariant map of $X$ into $G(M, p, q)$. Consider the following diagram, in which the vertical arrows represent projections.

$$
\begin{aligned}
\quad X \stackrel{\psi}{\longrightarrow} G(M, p, q) \stackrel{\phi}{\longrightarrow} G\left(R^{\infty}, p, p\right) \\
\underset{\downarrow}{\downarrow} \stackrel{\pi}{p}_{p} \stackrel{\hat{\psi}}{\longrightarrow} G(M, p, q) / \pi_{p} \stackrel{\hat{\phi}}{\longrightarrow} G\left(R^{\infty}, p, p\right) / \pi_{p}
\end{aligned}
$$

If $H^{i}(X)=0$ for $0<i \leqq N(M, p, q)$, then it follows from the naturality of 
the spectral sequence for a covering that $(\hat{\phi} \hat{\psi})^{*}$ is a monomorphism in degrees less than or equal to $N(M, p, q)+1$, contradicting the fact that $\hat{\phi}^{*}=0$ in degrees greater than $N(M, p, q)$. This proves the first part of the theorem.

Now suppose that $X$ is a $\mathbf{Z}_{p}$-orientable $m$-manifold. Observe that $\psi$ restricts to an equivariant map of $X-A(f, q)$ into $G(M, p, q)$ and that we may assume $X-A(f, q)$ is path connected. By the above argument there must be some $j, 0<j \leqq N(M, p, q)$, such that $H^{j}(X-A(f, q)) \neq 0$, and hence

$$
H_{j}(X-A(f, q)) \neq 0 .
$$

By Alexander Duality, $H^{m-j}(X, A(f, q)) \neq 0$. Similarly $H_{j}(X)=0$ implies $H^{m-j}(X)=0$, so by the exact cohomology sequence

$$
\bar{H}^{m-j-1}(A(f, q)) \neq 0 .
$$

By the argument which appears in [6], this is enough to prove that the covering dimension of $A(f, q)$ is greater than or equal to $m-N(M, p, q)-1$.

3. Proof of Theorem 4. First we remark that the case $p=q$ is particularly simple since $G\left(R^{n}, p, p\right)=\left(R^{n}\right)^{p}-\Delta \simeq S^{n(p-1)-1}$, and so $N\left(R^{n}, p, p\right) \leqq$ $n(p-1)-1$. The case $q=2$ appears in [4]. In general, we proceed as follows. The standard strong deformation retraction of $R^{n p}-\{0\}$ onto $S^{n p-1}$ restricts to a strong deformation retraction of $G\left(R^{n}, p, q\right)$ onto its intersection with $S^{n p-1}$. Let $K(n, p, p-q)$ denote the complement of the image of $G\left(R^{n}, p, q\right)$ under this deformation. We let $k=p-q$ and note that $K(n, p, k)$ is the union of spheres of dimension $n(k+1)-1$. Our method of bounding $N\left(R^{n}, p, q\right)$ will be the rather crude one of bounding $H^{*} G\left(R^{\infty}, p, q\right) / \mathbf{Z}_{p}$. In general we will do this by finding a lower bound for $H_{*} K(n, p, k)$ using the Mayer-Vietoris sequence and then applying Alexander Duality in the ( $n p$ - 1)-sphere.

First we need some notation for the pieces of $K(n, p, k)$ to which we will apply Mayer-Vietoris. Let $I=\left(i_{1}, \ldots, i_{j}\right), j \leqq k$, denote any $j$-tuple of integers with $0<i_{1}<i_{2}<\cdots<i_{j} \leqq p$. We define the length of $I$ to be $j$ and denote it by $l(I)$. We also permit $I$ to be empty and in this case define $l(I)=0$. Now let $m$ be any positive integer less than or equal to $p-k$ and define

$W(I, k, m)=\left\{\left(x, x, \ldots, x, y_{1}, x, x, \ldots, x, y_{2}, x, x, \ldots, x, y_{k}, x, x, \ldots, x\right) \mid\right.$

$x \in R^{n}, y_{s}$ occurs in the $i_{s}$ th place for $s=1,2, \ldots, j$,

and there are $m x$ 's between $y_{j}$ and $\left.y_{j+1}\right\}$.

That is, the coordinates which are not specified to be equal to other coordinates occur in places $i_{1}, i_{2}, \ldots, i_{j}, i_{j}+m+1$, and beyond. By abuse of notation we write the sequence $x, x, \ldots, x\left(\alpha_{1}\right.$ terms $)$ as $x^{\alpha_{1}}$. A typical point in $W(I, k, m)$ looks like

$$
\left(x^{\alpha_{1}} y_{1} x^{\alpha_{2}} y_{2} \cdots x^{\alpha_{j}} y_{j} x^{m} y_{j+1} x^{l_{1}} y_{j+2} x^{l_{2}} \cdots y_{k} x^{l_{k-j}}\right),
$$

where $\alpha_{1}+\cdots+\alpha_{j}+m+l_{1}+\cdots+l_{k-j}=p-k=q$. We note that the 
$\alpha_{i}^{\prime}$ 's are determined by $I$ and ignore them. Observe that $W(I, k, m)$ is a union of equatorial $(n(k+1)-1)$-spheres in $S^{n p-1}$. We assume that $q \geqq \frac{1}{2}(p+1)$.

Lemma 1. $\left[\cup_{i=0}^{m} W(I, k, i)\right] \cap W(I, k, m+1)=W(I, k-1, m+1)$.

Proof. Observe that a point is in the left-hand side if and only if $y_{j+1}=x$. Therefore $y_{j+2}, \ldots, y_{k}$ can be relabeled $y_{j+1}, \ldots, y_{k-1}$.

Lemma 2. $H_{i} W(I, k, m)=0$ if $0<i<n+k-1$.

Proof. The proof is by induction on $k$ and for fixed $k$ by downward induction on $l(I)$. The lemma is true for $k=0$ since $W(I, 0, m)$ is an $(n-1)$ sphere. Fix $k$ and assume that the lemma is true with $k-1$ replacing $k$. The induction on $l(I)$ starts with $l(I)=k$. In this case $W(I, k, m)$ is an $(n(k+1)-1)$-sphere, so the lemma is true. Now suppose that $l(I)=R-1$ and that the lemma is true for all $I$ with $k \geqq l(I) \geqq R$. A point

$$
x^{\alpha_{1}} y_{1} \cdots y_{R-1} x^{m}\left(y_{R} x^{l_{1}} \cdots x^{l_{R-1-k}}\right)
$$

can be rewritten as

$$
x^{\alpha_{1}} y_{1} \cdots y_{R} x^{l_{1}}\left(y_{R+1} \cdots x^{l_{R-1-k}}\right),
$$

so we have $W(I, k, m)=\cup_{t} W(J, k, t)$, where $t$ varies from 0 to some number $s$ determined by $I, k$, and $m$. Since $l(J)>l(I), H_{i} W(J, k, r)=0$ for $0<i$ $<n+k-1$ and all $r$ by induction. Now we assume that $H_{i}\left(\cup_{t=0}^{r} W(J, k, t)\right)$ $=0$ for $0<i<n+k-1$ and show that $H_{i}\left(\cup_{t=0}^{r+1} W(J, k, t)\right)=0$ for $0<i$ $<n+k-1$. By Lemma 1 the Mayer-Vietoris sequence is

$$
\begin{aligned}
\cdots & \rightarrow H_{i}\left(\bigcup_{t=0}^{r} W(J, k, t)\right) \oplus H_{i} W(J, k, r+1) \rightarrow H_{i}\left(\bigcup_{t=0}^{r+1} W(J, k, t)\right) \\
& \rightarrow H_{i-1} W(J, k-1, r+1) \rightarrow \cdots
\end{aligned}
$$

in which the left side is 0 for $0<i<n+k-1$ by the inductions on $r$ and on $l(I)$ and the right side is 0 for $0<i<n+k-1$ by the induction on $k$. Therefore $H_{i} W(I, k, m)=H_{i}(W(J, k, t))=0$ for $0<i<n+k-1$.

Remark. Note that the second half of this proof shows that for any sequence $J$, if $H_{i} W(J, k, t)=0$ for $0<i<n+k-1$ and all $t$, then $H_{i} \cup_{t=0}^{s} W(J, k, t)=0$ for $0<i<n+k-1$, for any $s$.

Proof of Theorem 4. First we note that $K(n, p, p-q)=\cup_{t=0}^{p} W(\varnothing, k, t)$, so by the above remark $H_{i} K(n, p, p-q)=0$ for $0<i<n+p-q-1$. By the remarks at the beginning of this section

$$
H^{i} G\left(R^{n}, p, q\right) \cong H^{i}\left(S^{n p-1}-K(n, p, p-q)\right),
$$

which is in turn isomorphic to $H_{n p-1-i}\left(S^{n p-1}, K(n, p, p-q)\right)$ by Alexander Duality. Then by the exact sequence

$$
\begin{aligned}
\cdots & \rightarrow H_{n p-1-i}\left(S^{n p-1}\right) \rightarrow H_{n p-1-i}\left(S^{n p-1}, K(n, p, p-q)\right) \\
& \rightarrow H_{n p-2-i}(K(n, p, p-q)) \rightarrow \cdots
\end{aligned}
$$

we have that $H^{i} G\left(R^{n}, p, q\right)=0$ for $i>(n-1)(p-1)+q-2$. This is suf- 
ficient, by the argument in [4], for example, to conclude that

$$
H^{i}\left(G\left(R^{n}, p, q\right) / \mathbf{Z}_{p}\right)=0 \text { for } i>(n-1)(p-1)+q-2 .
$$

4. An example. In some situations these results are best possible ones. For example, in [6] Munkholm gives an example for each odd $p$ and each $m$ of a $\pi_{p}$-action on $S^{m(p-1)-1}$ and a map from $S^{m(p-1)-1}$ to $R^{m}$ such that no entire orbit is sent to the same point in $R^{m}$. Our Theorem 1 states that there is an orbit in which $p-1$ points are sent to the same point. This example shows that in the case $q=p-1$ one can have $H^{i}(X)=0$ for $0<i<(n-1)$ $\cdot(p-1)+q-1$ with $A(f, q+1)=\varnothing$.

REMARK. We conjecture that Theorem 4 and hence Theorems 1 and 2 are true without the restriction $q \geqq \frac{1}{2}(p+1)$, although it is not hard to see that Lemma 1 and hence our method of proof break down if $q<\frac{1}{2}(p+1)$. The difficulty can be seen in the case $I=\varnothing, p=5, q=2, m=0$. Lemma 1 then says $W(\varnothing, 3,0) \cap W(\varnothing, 3,1)=W(\varnothing, 2,1)$. However a point of the form $(x, z, z, y, x)$ is in the left-hand side but not the right.

\section{REFERENCES}

1. K. Borsuk, Drei Sätze über die n-dimensional Euklidische Sphäre, Fund. Math. 20 (1933), 177-190.

2. D. G. Bourgin, On some separation and mapping theorems, Comment. Math. Helv. 29 (1955), 199-214. MR 17, 289.

3. F. Cohen and J. Connett, $A$ coincidence theorem related to the Borsuk-Ulam theorem, Proc. Amer. Math. Soc. 44 (1974), 218-220.

4. F. Cohen and E. L. Lusk, Coincidence point results for spaces with free $\mathbf{Z}_{p}$-actions, Proc. Amer. Math. Soc. 49 (1975), 245-252.

5. E. Fadell and L. Neuwirth, Configuration spaces, Math. Scand. 10 (1962), 111-118. MR 25 \#4537.

6. H. J. Munkholm, Borsuk-Ulam type theorems for proper $\mathbf{Z}_{p}$-actions on $(\bmod p$ homology) $n$ spheres, Math. Scand. 24 (1969), 167-185 (1970). MR 41 \#2672.

7. C.-T. Yang, Continuous functions from spheres to euclidean spaces, Ann. of Math. (2) 62 (1955), 284-292. MR 17, 289.

Department of Mathematics, Northern Illinois University, DeKalb, Illinois 60115 